\title{
Non Surgical Treatment of Chronic Anal Fissure: One year's experience with GTN paste
}

\author{
R AZIZ FU DIN M SHOAIB M KAMRAN \\ Department of Surgery, Fatima Jinnah Medical College/ Sir Ganga Ram Hospital, Lahore \\ Correspondence: Dr. Rizwan Aziz, Senior Registrar Surgery
}

\begin{abstract}
Objective: Assessment of the efficacy of topical $0.2 \%$ glyceryl trinitrate (GTN) paste in the treatment of chronic anal fissure. Patients and Methods: Sixty two patients were treated with $0.2 \%$ GTN paste and reviewed at 3, 6 and 12 weeks interval to assess efficacy and side effects. Results: At 6 weeks, $72 \%$ of patients were treated successfully. In $28 \%$ of cases treatment was unsuccessful. At 12 weeks follow up there was no early recurrence. The prevalence of headaches was $70 \%$. Conclusions: The use of GTN induces rapid healing of chronic anal fissures with a $72 \%$ healing rate in this study. But balance is required between fissure healing and headache intolerance.
\end{abstract}

Key words: Anal fissure, Glyceryl Trinitrate (GTN)

Anal fissure is an elongated ulcer in the long axis of anal canal. It may be acute or chronic. Chronic fissures-in-ano do not usually respond to dietary advice alone. In chronic anal fissure there is spasm of involuntary musculature of internal sphincter. The aim of treatment is to alleviate sphincter hypertonia and improve blood flow to the ulcerated area ${ }^{1}$. Lateral sphincterotomy permanently lowers resting anal pressure and in doing so aids the healing of anal fissures. It may, however, be associated with minor temporary or permanent alterations in the control of flatus, mucus and occasionally stool in up to $35 \%$ of patients ${ }^{2}$. This has led to alternative therapeutic approaches, in particular, using topical agents such as botulinum toxin, calcium channel blockers and glyceryl trinitrate (GTN).

The most widely used topical agent is GTN which is metabolized to nitric oxide and leads to sphincter muscle relaxation and reduction in the maximum anal resting pressure. This eliminates the risk of permanent anal incontinence associated with surgery ${ }^{3}$.

\section{Patients and methods:}

Sixty two patients with chronic anal fissure were included in the study. Chronicity was determined by a history longer than 3 months and/or the presence on examination of a sentinel tag at the base of the fissure. Patients with inflammatory bowel disease, pregnant women and patients taking nitrates for other conditions were excluded.

At presentation, a pain score $(0-10)$ was established as well as a symptom score (0-3) with one point each for bleeding, discharge and itching. The patients were started on a course of $0.2 \%$ GTN paste which was applied digitally peri- and intra-anally three times daily, initially for 6 weeks. The amount applied was the smallest amount that could be rubbed into the anal area. All patients were reviewed at 3, 6, and 12 weeks to assess pain and symptoms scores and to assess fissure healing, complications and compliance. Patients that did not respond to treatment or who were unable to comply with the treatment were offered a lateral anal sphincterotomy.

\section{Results:}

The mean age of patients was 40 years (range 17-80 years). The mean length of anal fissure history was 8 months (range 4-36 months). The mean pain score at presentation was 8 (range $2-10$ ).

The mean pain score at 3-week follow up was 3 with $20 / 62(32.2 \%)$ patients experiencing no pain. At 6 weeks, $28 / 62(45.16 \%)$ patients experienced no pain and $35 / 62$ $(56.45 \%)$ were pain free at 12 weeks. At the time of presentation 45 out of $62(72 \%)$ patients had two or more of the documented symptoms. At 3 weeks, 36 (58.06\%) patients were symptom free. At 3 month follow up this was $40(64.51 \%)$ patients. Overall, at 12 weeks following treatment with $0.2 \%$ GTN paste, $(72 \%)$ patients had a clinically healed ulcer (Fig 1). Four of these patients continued to experience pain in the absence of a fissure but were asymptomatic when reviewed at 18 weeks.

In $17(28 \%)$ patients, treatment with $0.2 \%$ GTN paste was unsuccessful. Of these, 11 patients were not compliant, 3 did not respond to treatment, 1 became pregnant during treatment and 2 did not attend follow-up. At 6 month follow-up, the $72 \%$ patients successfully treated all remained symptom free with healed fissures. There were no fissure recurrences.

\section{Discussion:}

Topical GTN paste is an effective alternative to surgery in the treatment of chronic fissures-in-ano. At a concentration of $0.2 \%$, GTN has been shown to be better than placebo $(68 \%$ vs $8 \%)$ in healing chronic anal fissures. ${ }^{4}$ A crescendo dosage of GTN from $0.2 \%$ to $0.6 \%$, by $0.1 \%$ weekly increments, was associated with a consistently lower pain score and a better healing rate $(70 \%$ vs $64 \%)$ compared with the $0.2 \%$ treatment group but this did not reach statistical significance ${ }^{5}$.

Most data to support the use of topical GTN has been based on the application of $0.2 \%$ GTN. Only one small uncontrolled study has previously assessed the use of topical $0.5 \%$ GTN ointment in the treatment of anal fissures ${ }^{6}$. This study, however, included patients with Crohn's disease and thrombosed external piles; indeed only 
12 patients in this series had primary fissures-in-ano. Curiously, headaches were reported in only 7 of the 20 patients in Gorfine's (1995) study despite use of the $0.5 \%$ GTN paste 4 times a day. ${ }^{6}$ More recently, a randomised controlled trial that included a treatment arm of weekly increases in GTN ointment concentration of $0.1 \%$ - starting at $0.2 \%$ and progressing to $0.6 \%$ over an 8 week period showed no statistically significant difference in outcome compared with a fixed $0.2 \%$ concentration5. Our series shows that up to $73 \%$ of patients with chronic anal fissures can be successfully treated using topical $0.2 \%$ GTN paste as a primary measure. The rapid symptomatic improvement with $0.2 \%$ GTN compares favourably with the use of other regimens of GTN or weekly increments in GTN concentration. Whilst there is little difference in the overall outcome of treatment there does appear to be a trend towards a better outcome with the use of higher doses ( $73 \%$ with $0.5 \%$ vs $70 \%$ using incremental doses vs $64 \%$ with $0.2 \%)^{4,5}$. The use of $0.5 \%$ GTN is associated with an $70 \%$ incidence of headaches. In five patients (8\%) headache intolerance was the cause for non-compliance or dis-continuation of treatment. This compares with a headache incidence of $58 \%$, when using $0.2 \%$ GTN ointment, and 3\% non-compliance due to this side effect ${ }^{4}$. Of note is the minimal difference in the findings at 6 and 12 weeks follow-up. Symptom scores were nearly identical with partially healed ulcers at 6 weeks completing the healing process within 12 weeks. It would appear that compliant patients that respond to treatment do so within 6 weeks or probably not at all. This suggests that failure to respond to topical GTN at 6 weeks may be an indication for an alternative therapeutic approach.

Early recurrence does not appear to be a problem. Long- term recurrence and its' treatment options are currently the subject of a study in this hospital. A recent study has shown 11 symptomatic relapses in 44 patients treated with topical $0.2 \%$ GTN 3 months from termination of treatment ${ }^{7}$. From a quarter to a third of patients in another study experienced recurrence at a median follow up of 9 months 5 .

Short-term follow up suggests that there is little justification in using a higher dose of GTN paste. Longer follow up series with various doses, however, are required in order to establish the optimal dosage. Standardisation of the method of application and the amount of ointment applied are also required to validate metanalysis in the future.

\section{References:}

1. Watson SJ, Kamm MA, Nicholls RJ, Phillips RKS. Topical glyceryl trinitrate in the treatment of chronic anal fissure. British Journal of Surgery 1996 83; 771-5

2. Khubchandani IT, Reed JF. Sequelae of internal sphincterotomy for chronic fissure in ano. British Journal of Surgery $198976 ; 431-4$

3. Schouten WR, Briel JW, Auwerda JJA. Relationship between anal pressure and anodermal blood flow: the vascular pathogenesis of anal fissure. Gut 1994 34; S25

4. Lund JN, Scholefield JH. A randomised, prospective, double blind, placebo controlled trial of glyceryl trinitrate ointment in treatment of anal fissure. Lancet 1997 349; 11-4

5. Carapeti EA, Kamm MA, McDonald PJ, Chadwick SJD, Melville D, Phillips RKS. Randomised controlled trial shows that glyceryl trinitrate heals anal fissures, higher doses are not more effective, and there is a high recurrence rate. 1999 Gut $44 ; 727-30$

6. Gorfine SR. Treatment of benign anal disease with topical nitroglycerin. Diseases of the Colon \& Rectum 1995 38; 453-7

7. Lund JN, Scholefield JH. Follow-up of patients with chronic anal fissure treated with topical glyceryl trinitrate. Lancet $1998352 ; 1681$ 